\title{
Wirtschaftlichkeitskontrolle in der Krankenversicherung
}

\author{
Die nachstehenden Überlegungen sind die Zusammenfassung eines Gutachtens, \\ das für den Verein Ethik und Medizin erstellt wurde.*
}

Ueli Kieser

1 Bundesblatt 2011; 2524

* Im Gutachten finden sich zu den im vorliegenden Text nur knapp zusammengefassten Überlegungen weitere Ausführungen und Belege. physicianprofiling.ch/ KieserGutachten2014.pdf

Korrespondenz: Prof. Dr. iur. Ueli Kieser Rechtsanwalt Kieser Senn Partner Ulrichstrasse 14 CH-8032 Zürich ukieser[at]kspartner.ch
Leistungen in der Krankenversicherung haben wirksam, zweckmässig und wirtschaftlich zu sein - so legt es Art. 32 Abs. 1 KVG fest. Das Gesetz hält ferner in Art. 32 Abs. 2 KVG fest, dass die Wirtschaftlichkeit der Leistungen periodisch überprüft wird. Dazu legt Art. 56 KVG fest, dass sich die Leistungserbringenden (z. B. Ärztinnen und Ärzte) in ihren Leistungen auf das Mass zu beschränken haben, das im Interesse der Versicherten liegt und für den Behandlungszweck erforderlich ist. Wie diese Wirtschaftlichkeitskontrolle durchzuführen ist, ist seit je umstritten.

Art. 56 Abs. 6 KVG bestimmt, dass sich Leistungserbringende und Versicherer vertraglich auf eine Methode zur Kontrolle der Wirtschaftlichkeit festlegen. Dazu ist es bis zum heutigen Zeitpunkt trotz verschiedener Bemühungen und trotz intensiver Arbeit - noch nicht gekommen. Die Beteiligten finden sich heute also gewissermassen in einem Schwebezustand. Dies soll Anlass sein, um nachstehend auf einige zentrale Grundsätze hinzuweisen, welche jedenfalls bei einer Wirtschaftlichkeitskontrolle berücksichtigt werden müssen.

Die Wirtschaftlichkeitskontrolle in der Krankenversicherung muss in jedem Fall die allgemeinen Zielsetzungen der Krankenversicherung berücksichtigen. Diese will eine qualitativ hochstehende gesundheitliche Versorgung zu möglichst günstigen Kosten sicherstellen. Das primäre Ziel der Krankenversicherung besteht also darin, eine überdurchschnittliche - eben eine qualitativ hochstehende gesundheitliche Versorgung zu erreichen. Diesem primären Ziel der Krankenversicherung ist die Kostenfrage nachgelagert; dies wird dadurch zum Ausdruck gebracht, dass die medizinische Versorgung (nur, aber immerhin) zu möglichst günstigen Kosten erfolgen soll.

Bei der Konkretisierung der Kontrollmethode dürfen ausschliesslich sachlich zutreffende Kriterien verwendet werden. Es müssen solche Kriterien sein, welche einer objektiven Überprüfung zugänglich sind und welche zudem geeignet sind, die Wirtschaftlichkeitsprüfung effektiv vornehmen zu können. Als im Nationalrat über die Konkretisierung der Wirtschaftlichkeitskontrollmethode debattiert wurde, wurde - einzig - festgehalten, dass jedenfalls die Morbidität der jeweiligen Patientenkollektive abgebildet werden soll [1].
Es hat sich in der bisherigen Praxis weitgehend die Durchschnittskostenüberprüfung durchgesetzt. Eine solche Kontrollmethode ist nicht an sich verfehlt. Freilich setzt sie voraus, dass in statistischer Hinsicht unanfechtbare Kriterien herangezogen werden. Wie die bisherigen Diskussionen zeigen, stehen im heutigen Zeitpunkt die entsprechenden valablen Daten noch nicht unbedingt zur Verfügung. Es kommt die Schwierigkeit hinzu, dass die Vergleichsgruppe zutreffend zusammengesetzt sein muss. Gerade dem letztgenannten Aspekt kommt ein absolut zentrales Gewicht zu. Dabei muss einerseits darauf geachtet werden, dass die Vergleichsgruppe nicht zu klein ausfällt. Anderseits ist zu berücksichtigen, dass die Vergleichsgruppe die Voraussetzungen spiegelt, die für den zu überprüfenden einzelnen Leistungserbringer gelten. Diesbezüglich ist etwa zu berücksichtigen, dass der Grundsatz der Homogenität der Kostenstruktur der Praxis gewahrt bleibt und dass in der Vergleichsgruppe tatsächlich dieselben Behandlungen vorgenommen werden.

Angesichts der heute nach wie vor bestehenden Schwierigkeit, verlässliche statistische Daten beibringen zu können, mag ein Ausweg darin bestehen, dass verschiedene Untersuchungs- und Überprüfungsmethoden gleichzeitig angewendet werden. So ist allenfalls eher möglich, eine Validierung der Überprüfung zu ermöglichen. Im heutigen Zeitpunkt steht jedenfalls fest, dass eine nach bisheriger Überprüfungsweise vorgenommene Kontrolle nicht mehr ausreicht, eine allfällige Unwirtschaftlichkeit der Behandlung feststellen zu können. Es liegen mittlerweile greifbare Einwände vor, die nahelegen, überhaupt nicht mehr bzw. nicht mehr einzig auf die bisherigen Methoden (Rechnungstellerstatistik RSS bzw. ANOVA-Methode) abzustellen. Damit ist heute in einer umfassenderen und überblicksmässigen Gesamtprüfung festzustellen, ob allenfalls eine Unwirtschaftlichkeit der Behandlung vorliegt. Besonders dringend ist es ferner, dass überzeugende und nachprüfbare neue Methoden bald entwickelt werden, um die im heutigen Zeitpunkt bestehende Unsicherheit beheben zu können. Eine vertragliche Einigung zwischen Ärzteschaft und Krankenversicherern wird wohl erst Sinn machen, wenn neue und allseitig anerkannte Prüfmethoden bestimmt worden sind. 